\title{
Graph Treewidth and Geometric Thickness Parameters
}

\author{
Vida Dujmović ${ }^{1, \star}$ and David R. Wood ${ }^{2, \star \star}$ \\ 1 School of Computer Science, Carleton University, Ottawa, Canada \\ vida@scs. carleton.ca \\ 2 Departament de Matemàtica Aplicada II, Universitat Politècnica de Catalunya, \\ Barcelona, Spain \\ david. wood@upc.edu
}

\begin{abstract}
Consider a drawing of a graph $G$ in the plane such that crossing edges are coloured differently. The minimum number of colours, taken over all drawings of $G$, is the classical graph parameter thickness $\theta(G)$. By restricting the edges to be straight, we obtain the geometric thickness $\bar{\theta}(G)$. By further restricting the vertices to be in convex position, we obtain the book thickness bt $(G)$. This paper studies the relationship between these parameters and the treewidth of $G$. Let $\theta\left(\mathcal{T}_{k}\right) / \bar{\theta}\left(\mathcal{I}_{k}\right)$ / bt $\left(\mathcal{T}_{k}\right)$ denote the maximum thickness / geometric thickness / book thickness of a graph with treewidth at most $k$. We prove that:

$-\theta\left(\mathcal{T}_{k}\right)=\bar{\theta}\left(\mathcal{T}_{k}\right)=\lceil k / 2\rceil$, and

- bt $\left(\mathcal{T}_{k}\right)=k$ for $k \leq 2$, and $\operatorname{bt}\left(\mathcal{T}_{k}\right)=k+1$ for $k \geq 3$.

The first result says that the lower bound for thickness can be matched by an upper bound, even in the more restrictive geometric setting. The second result disproves the conjecture of Ganley and Heath [Discrete Appl. Math. 2001] that $\mathrm{bt}\left(\mathcal{T}_{k}\right)=k$ for all $k$. Analogous results are proved for outerthickness, arboricity, and star-arboricity.
\end{abstract}

\section{Introduction}

Partitions of the edge set of a graph $G$ into a small number of 'nice' subgraphs is in the mainstream of graph theory. For example, in a proper edge colouring, the subgraphs of the partition are matchings. When the subgraphs are required to be planar (respectively, acyclic), then the minimum number of subgraphs in a partition of $G$ is the thickness (arboricity) of $G$. Thickness and arboricity are classical graph parameters that have been studied since the early 1960's. The first results in this paper concern the relationship between treewidth and parameters such as thickness and arboricity. Treewidth is a more modern graph parameter which is particularly important in structural and algorithmic graph theory. For each of thickness and arboricity (and other related parameters), we prove tight bounds on the minimum number of subgraphs in a partition of a graph with treewidth $k$. These introductory results are presented in Section 2

* Supported by NSERC postdoctoral grant.

** Supported by the Government of Spain grant MEC SB-2003-0270, and by projects MCYT-FEDER BFM2003-00368 and Gen. Cat 2001SGR00224. 
The main results of the paper concern partitions of graphs with an additional geometric property. Namely, that there is a drawing of the graph, and each subgraph in the partition is drawn without crossings. This type of drawing has applications in graph visualisation (where each plane subgraph is coloured by a distinct colour), and in multilayer VLSI (where each plane subgraph corresponds to a set of wires that can be routed without crossings in a single layer). When there is no restriction on the edges, the minimum number of plane subgraphs, taken over all drawings of $G$, is again the thickness of $G$. By restricting the edges to be straight, we obtain the geometric thickness of $G$. By further restricting the vertices to be in convex position, we obtain the book thickness of G. Our main results precisely determine the maximum geometric thickness and maximum book thickness of all graphs with treewidth $k$. We also determine the analogous value for a number of other related parameters.

The paper is organised as follows. Section 3 formally introduces all of the geometric parameters to be studied. Section 4 states our main results. The proofs of our two main theorems are presented in Sections 5 and 6 . The remaining proofs are in the full version of the paper 6 .

\section{Abstract Graph Parameters}

We consider graphs $G$ that are simple, finite, and undirected. Let $V(G)$ and $E(G)$ denote the vertex and edge sets of $G$. For $A, B \subseteq V(G)$, let $G[A ; B]$ denote the bipartite subgraph of $G$ with vertex set $A \cup B$ and edge set $\{v w \in E(G): v \in$ $A, w \in B\}$. A graph parameter is a function $f$ such that $f(G) \in \mathbb{N}$ for all graphs $G$. For a graph class $\mathcal{G}$, let $f(\mathcal{G}):=\max \{f(G): G \in \mathcal{G}\}$. If $f(\mathcal{G})$ is unbounded, then let $f(\mathcal{G}):=\infty$.

The thickness of a graph $G$, denoted by $\theta(G)$, is the minimum number of planar subgraphs that partition $E(G)$ (see [1]). A graph is outerplanar if it has a plane drawing with all the vertices on the boundary of the outerface. The outerthickness of a graph $G$, denoted by $\theta_{\mathrm{o}}(G)$, is the minimum number of outerplanar subgraphs that partition $E(G)$ (see [8]). The arboricity of a graph $G$, denoted by a $(G)$, is the minimum number of forests that partition $E(G)$. [12] proved that $\mathrm{a}(G)=\max \left\{\left\lceil\frac{|E(H)|}{|V(H)|-1}\right\rceil: H \subseteq G\right\}$. A star-forest is graph in which every component is a star. The star-arboricity of a graph $G$, denoted by $\operatorname{sa}(G)$, is the minimum number of star-forests that partition $E(G)$ (see [1]). Thickness, outerthickness, arboricity and star-arboricity are always within a constant factor of each other (see [6]).

In the remainder of this section we determine the maximum value of each of the above four parameters for graphs of treewidth $k$. A set of $k$ pairwise adjacent vertices in a graph $G$ is a $k$-clique. For a vertex $v$ of $G$, let $N_{G}(v):=$ $\{w \in V(G): v w \in E(G)\}$ and $N_{G}[v]:=N_{G}(v) \cup\{v\}$. We say $v$ is $k$-simplicial if $N_{G}(v)$ is a $k$-clique. A $k$-tree is a graph $G$ such that either $G$ is (isomorphic to) the complete graph $K_{k}$, or $G$ has a $k$-simplicial vertex $v$ and $G \backslash v$ is a $k$-tree. The treewidth of a graph $G$ is the minimum $k \in \mathbb{N}$ such that $G$ is a spanning subgraph of a $k$-tree. Let $\mathcal{T}_{k}$ denote the class of graphs with 
treewidth at most $k$. Many families of graphs have bounded treewidth. $\mathcal{T}_{1}$ is the class of forests. Graphs in $\mathcal{T}_{2}$ are obviously planar - a 2-simplicial vertex can always be drawn near the edge connecting its two neighbours. Graphs in $\mathcal{T}_{2}$ are characterised as those with no $K_{4}$-minor, and are sometimes called series-parallel.

Theorem 1. $\theta\left(\mathcal{T}_{k}\right)=\lceil k / 2\rceil$

Proof. The upper bound immediately follows from a more general result by [4. Now for the lower bound. The result is trivial if $k \leq 2$. Assume $k \geq 3$. Let $\ell:=\lceil k / 2\rceil-1$. Let $G$ be the $k$-tree obtained by adding $2 \ell^{k}+1 k$-simplicial vertices adjacent to each vertex of a $k$-clique. Suppose that $\theta(G) \leq \ell$. In the corresponding edge $\ell$-colouring of $G$, consider the vector of colours on the edges incident to each $k$-simplicial vertex. There are $\ell^{k}$ possible colour vectors. Thus there are at least three $k$-simplicial vertices $x, y, z$ with the same colour vector. At least $\lceil k / \ell\rceil \geq 3$ of the $k$ edges incident to $x$ are monochromatic. Say these edges are $x a, x b, x c$. Since $y$ and $z$ have the same colour vector as $x$, the $K_{3,3}$ subgraph induced by $\{x a, x b, x c, y a, y b, y c, z a, z b, z c\}$ is monochromatic. Since $K_{3,3}$ is not planar, $\theta(G) \geq \ell+1=\lceil k / 2\rceil$. Therefore $\theta\left(\mathcal{T}_{k}\right) \geq\lceil k / 2\rceil$.

The proofs of the following two results are similar to that of Theorem 1 and can be found in the full version of the paper [6].

Theorem 2. $\theta_{o}\left(\mathcal{T}_{k}\right)=\mathrm{a}\left(\mathcal{T}_{k}\right)=k$

Theorem 3. $\operatorname{sa}\left(\mathcal{T}_{k}\right)=k+1$

\section{Geometric Parameters}

For our purposes, a drawing of a graph represents the vertices by a set of points in the plane in general position (no three collinear), and represents each edge by a simple closed curve between its endpoints, such that the only vertices that an edge intersects are its own endpoints. Two edges cross if they intersect at some point other than a common endpoint. A graph drawing with no crossings is plane. A plane drawing in which all the vertices are on the outerface is outerplane.

The thickness of a graph drawing is the minimum $k \in \mathbb{N}$ such that the edges of the drawing can be partitioned into $k$ plane subgraphs; that is, each edge is assigned one of $k$ colours such that monochromatic edges do not cross. Any planar graph can be drawn with its vertices at prespecified locations [9, 13]. Thus a graph with thickness $k$ has a drawing with thickness $k$ [9. However, in such a representation the edges may be highly curved. This motivates the notion of geometric thickness.

A drawing of a graph is geometric if every edge is represented by a straight line-segment. The geometric thickness of a graph $G$, denoted by $\bar{\theta}(G)$, is the minimum $k \in \mathbb{N}$ such that there is a geometric drawing of $G$ with thickness $k$. [10] first defined geometric thickness under the name of real linear thickness, 
and it has also been called rectilinear thickness. By the Fáry-Wagner theorem, a graph has geometric thickness one if and only if it is planar.

We generalise the notion of geometric thickness as follows. The outerthickness of a graph drawing is the minimum $k \in \mathbb{N}$ such that the edges of the drawing can be partitioned into $k$ outerplane subgraphs. The arboricity and star-arboricity of a graph drawing are defined similarly, where it is respectively required that each subgraph be a plane forest or a plane star-forest. Again a graph with outerthickness /arboricity / star-arboricity $k$ has a drawing with outerthickness / arboricity / star-arboricity $k$ 9, 13. The geometric outerthickness / geometric arboricity / geometric star-arboricity of a graph $G$, denoted by $\overline{\theta_{\mathrm{o}}}(G) / \overline{\mathrm{a}}(G)$ / $\overline{\mathrm{sa}}(G)$, is the minimum $k \in \mathbb{N}$ such that there is a geometric drawing of $G$ with outerthickness / arboricity / star-arboricity $k$.

A geometric drawing in which the vertices are in convex position is called a book embedding. The book thickness of a graph $G$, denoted by bt $(G)$, is the minimum $k \in \mathbb{N}$ such that there is book embedding of $G$ with thickness $k$. Note that whether two edges cross in a book embedding is simply determined by the relative positions of their endpoints in the cyclic order of the vertices around the convex hull. One can think of the vertices as being ordered on the spine of a book and each plane subgraph being drawn without crossings on a single page. Book embeddings are ubiquitous structures with a variety of applications; see [5] for a survey with over 50 references. A graph has book thickness one if and only if it is outerplanar 2. A graph has a book thickness at most two if and only if it is a subgraph of a Hamiltonian planar graph [2] 15] proved that planar graphs have book thickness at most four.

The book arboricity / book star-arboricity of a graph $G$, denoted by ba $(G)$ / bsa $(G)$, is the minimum $k \in \mathbb{N}$ such that there is a book embedding of $G$ with arboricity / star-arboricity $k$. There is no point in defining "book outerthickness" since it would always equal book thickness.

\section{Main Results}

In this paper we determine the value of all of the geometric graph parameters defined in Section 3 for $\mathcal{T}_{k}$. The following theorem, which is proved in Section 6, is the most significant result in the paper. It says that the lower bound for the (abstract) thickness of $\mathcal{T}_{k}$ (Theorem 1) can be matched by an upper bound, even in the more restrictive setting of geometric thickness.

Theorem 4. $\bar{\theta}\left(\mathcal{T}_{k}\right)=\lceil k / 2\rceil$

We have the following theorem for the geometric outerthickness and geometric arboricity of $\mathcal{T}_{k}$. It says that the lower bounds for the outerthickness and arboricity of $\mathcal{T}_{k}$ can be matched by an upper bound on the corresponding geometric parameter. By the lower bound in Theorem [2, to prove Theorem [5] it suffices to show that $\overline{\mathrm{a}}\left(\mathcal{T}_{k}\right) \leq k$; we do so in [6]. 
Theorem 5. $\overline{\theta_{o}}\left(\mathcal{T}_{k}\right)=\overline{\mathrm{a}}\left(\mathcal{T}_{k}\right)=k$

We have the following theorem for the book thickness and book arboricity of $\mathcal{T}_{k}$.

Theorem 6. $\operatorname{bt}\left(\mathcal{T}_{k}\right)=\operatorname{ba}\left(\mathcal{T}_{k}\right)= \begin{cases}k & \text { for } k \leq 2 \\ k+1 & \text { for } k \geq 3\end{cases}$

This theorem gives an example of an abstract parameter that is not matched by its geometric counterpart. In particular, bt $\left(\mathcal{T}_{k}\right)>\theta_{\circ}\left(\mathcal{T}_{k}\right)=k$ for $k \geq 3$. Theorem 6 with $k=1$ was proved by 2 . That bt $\left(\mathcal{T}_{2}\right) \leq 2$ was independently proved by [14] and 3. Note that bt $\left(\mathcal{T}_{2}\right)=2$ since there are series parallel graphs that are not outerplanar, $K_{2,3}$ being the primary example. We prove the stronger result that ba $\left(\mathcal{T}_{2}\right)=2$ in [6. 7] proved that every $k$-tree has a book embedding with thickness at most $k+1$. It is easily seen that each plane subgraph is in fact a star-forest. Thus $\operatorname{bt}\left(\mathcal{T}_{k}\right) \leq \mathrm{ba}\left(\mathcal{T}_{k}\right) \leq \mathrm{bsa}\left(\mathcal{T}_{k}\right) \leq k+1$. We give an alternative proof of this result in [6]. [7] proved a lower bound of bt $\left(\mathcal{T}_{k}\right) \geq k$, and conjectured that $\mathrm{bt}\left(\mathcal{T}_{k}\right)=k$. Thus Theorem [6 refutes this conjecture. The proof is given in Section [5] where we construct a $k$-tree $G$ with bt $(G)>k$.

Finally observe that the upper bound of [7] mentioned above and the lower bound in Theorem 3 prove the following result for the star-arboricity of $\mathcal{T}_{k}$.

Theorem 7. $\operatorname{sa}\left(\mathcal{T}_{k}\right)=\overline{\mathrm{sa}}\left(\mathcal{T}_{k}\right)=\operatorname{bsa}\left(\mathcal{T}_{k}\right)=k+1$

\section{Book Thickness: Proof of Theorem $6(k \geq 3)$}

By the discussion in Section 4, it suffices to show that for all $k \geq 3$, there is a $k$-tree $G$ with book thickness bt $(G)>k$. Define $G$ by the following construction:

- Start with a $k$-clique $V_{1}$.

- Add $k(2 k+1) k$-simplicial vertices adjacent to each vertex in $V_{1}$; call this set of vertices $V_{2}$.

- For each vertex $v \in V_{2}$, choose three distinct vertices $x_{1}, x_{2}, x_{3} \in V_{1}$, and for each $1 \leq i \leq 3$, add four $k$-simplicial vertices adjacent to each vertex of the clique $\left(V_{1} \cup\{v\}\right) \backslash\left\{x_{i}\right\}$. Each set of four vertices is called an $i$-block of $v$. Let $V_{3}$ be the set of vertices added in this step.

Clearly $G$ is a $k$-tree. Assume for the sake of contradiction that $G$ has a book embedding with thickness $k$. Let $\left\{E_{1}, E_{2}, \ldots, E_{k}\right\}$ be the corresponding partition of the edges. For each ordered pair of vertices $v, w \in V(G)$, let the arc-set $V_{\widehat{v w}}$ be the list of vertices in clockwise order from $v$ to $w$ (not including $v$ and $w$ ). Say $V_{1}=\left(y_{1}, y_{2}, \ldots, y_{k}\right)$ in anticlockwise order. There are $k(2 k+1)$ vertices in $V_{2}$. Without loss of generality there are at least $2 k+1$ vertices in $V_{2} \cap V_{\widehat{y_{1} y_{k}}}$. Let $\left(v_{1}, v_{2}, \ldots, v_{2 k+1}\right)$ be $2 k+1$ vertices in $V_{2} \cap V_{\widehat{y_{1} y_{k}}}$ in clockwise order.

Observe that the $k$ edges $\left\{y_{i} v_{k-i+1}: 1 \leq i \leq k\right\}$ are pairwise crossing, and thus receive distinct colours, as illustrated in Figure 1(a). Without loss of generality, each $y_{i} v_{k-i+1} \in E_{i}$. As illustrated in Figure 1(b), this implies that 
$y_{1} v_{2 k+1} \in E_{1}$, since $y_{1} v_{2 k+1}$ crosses all of $\left\{y_{i} v_{k-i+1}: 2 \leq i \leq k\right\}$ which are coloured $\{2,3, \ldots, k\}$. As illustrated in Figure 1(c), this in turn implies $y_{2} v_{2 k} \in$ $E_{2}$, and so on. By an easy induction, we obtain that $y_{i} v_{2 k+2-i} \in E_{i}$ for all $1 \leq i \leq k$, as illustrated in Figure 1(d). It follows that for all $1 \leq i \leq k$ and $k-i+1 \leq j \leq 2 k+2-i$, the edge $y_{i} v_{j} \in E_{i}$, as illustrated in Figure 1(e). Finally, as illustrated in 1(f), we have:

If $q y_{i} \in E(G)$ and $q \in V_{v_{k-1} v_{k}+3}$, then $q y_{i} \in E_{i}$.
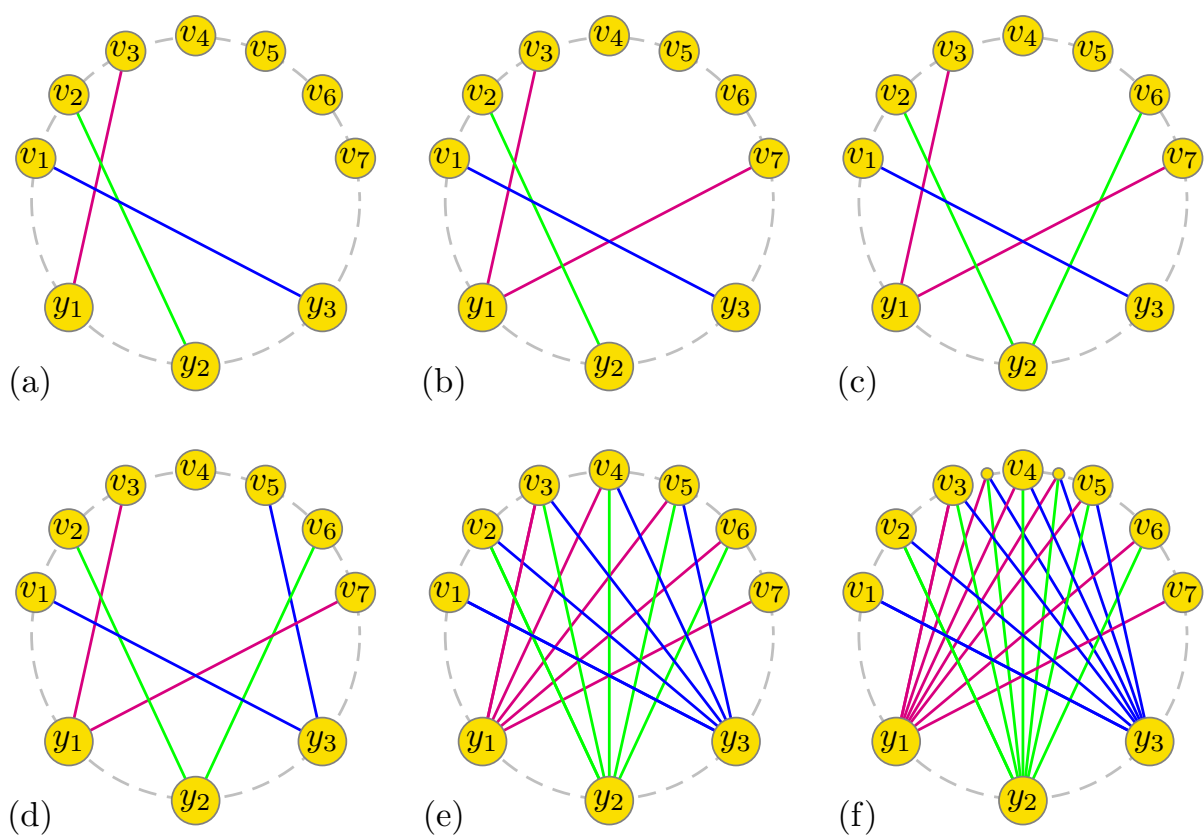

Fig. 1. Example in the proof of Theorem $[$ with $k=3$

Consider any of the twelve vertices $w \in V_{3}$ that are added onto a clique that contain $v_{k+1}$. Then $w$ is adjacent to $v_{k+1}$. Moreover, $w$ is in $V_{v_{k} v_{k+1}}$ or $V_{v_{k+1} v_{k}+2}$, as otherwise the edge $w v_{k+1}$ crosses $k$ edges of $G\left[\left\{v_{k-1}, v_{k+1}\right\} ; V_{1}\right]$ that are all coloured differently, which is a contradiction. By the pigeon-hole principle, one of $V_{v_{k} v_{k+1}}$ and $V_{v_{k+1} v_{k+2}}$ contains at least two vertices from two distinct $p$-blocks of $v_{k+1}$. Without loss of generality, $V_{\widehat{v_{k} v_{k+1}}}$ does. Let these four vertices be $(a, b, c, d)$ in clockwise order.

Each vertex in $\{b, c, d\}$ is adjacent to $k-1$ vertices of $V_{1}$. Not all of $b, c, d$ are adjacent to the same subset of $k-1$ vertices in $V_{1}$, as otherwise all of $b, c, d$ would belong to the same $p$-block. Hence each vertex in $V_{1}$ has a neighbour in $\{b, c, d\}$. By $(\star)$ the edges of $G\left[\{b, c, d\}, V_{1}\right]$ receive all $k$ colours. However, every edge in $G\left[\{b, c, d\} ; V_{1}\right]$ crosses the edge $a v_{k+1}$, implying that there is no colour available for $a v_{k+1}$. This contradiction completes the proof. 


\section{Geometric Thickness: Proof of Theorem 4}

The proofs of all of our upper bounds depend upon the following lemma.

Lemma 8. For every $k$-tree $G$, either:

(1) there is a (possibly empty) independent set $S \subseteq V(G)$ of $k$-simplicial vertices in $G$ such that $G \backslash S=K_{k}$, or

(2) there is a nonempty independent set $S \subseteq V(G)$ of $k$-simplicial vertices in $G$ and a vertex $v \in V(G) \backslash S$, such that:

(a) $G \backslash S$ is a $k$-tree,

(b) $v$ is k-simplicial in $G \backslash S$,

(c) for every vertex $w \in S$, there is exactly one vertex $u \in N_{G \backslash S}(v)$ such that $N_{G}(w)=N_{G \backslash S}[v] \backslash\{u\}$,

(d) every $k$-simplicial vertex of $G$ that is not in $S$ is not adjacent to $v$.

Proof. We proceed by induction on $|V(G)|$. If $|V(G)|=k$ then $G=K_{k}$ and property (1) is satisfied with $S=\emptyset$. If $|V(G)|=k+1$ then $G=K_{k+1}$ and property (1) is satisfied with $S=\{v\}$ for any vertex $v$. Now suppose that $|V(G)| \geq k+2$. Let $L$ be the set of $k$-simplicial vertices of $G$. Then $L$ is a nonempty independent set, and $G \backslash L$ is a $k$-tree. Moreover, the neighbourhood of each vertex in $L$ is a $k$-clique. If $G \backslash L=K_{k}$, then property (1) is satisfied with $S=L$. Otherwise, $G \backslash L$ has a $k$-simplicial vertex $v$. Let $S$ be the set of neighbours of $v$ in $L$. We claim that property (2) is satisfied. Now $S \neq \emptyset$, as otherwise $v \in L$. Since $G$ is not a clique and each vertex in $S$ is simplicial, $G \backslash S$ is a $k$-tree. Consider a vertex $w \in S$. Now $N_{G}(w)$ is a $k$-clique and $v \in N_{G}(w)$. Thus $N_{G}(w) \subseteq N_{G \backslash S}[v]$. Since $\left|N_{G}(w)\right|=k$ and $\left|N_{G \backslash S}[v]\right|=k+1$, there is exactly one vertex $u \in N_{G \backslash S}(v)$ for which $N_{G}(w)=N_{G \backslash S}[v] \backslash\{u\}$. Part (d) is immediate.

We now turn to the proof of Theorem 4 . The lower bound $\bar{\theta}\left(\mathcal{T}_{k}\right) \geq\lceil k / 2\rceil$ follows from the stronger lower bound $\theta\left(\mathcal{T}_{k}\right) \geq\lceil k / 2\rceil$ in Theorem 1 . The theorem is true for all 0 -, 1 - and 2 -trees since they are planar. To prove the upper bound $\bar{\theta}\left(\mathcal{T}_{k}\right) \leq\lceil k / 2\rceil$, it suffices to prove that $\bar{\theta}(2 k) \leq k$ for all $k \geq 2$. Let $I:=\{i,-i$ : $1 \leq i \leq k\}$.

Consider a geometric drawing of a $2 k$-tree $G$, in which the edges are coloured with $k$ colours. Let $v$ be a $2 k$-simplicial vertex of $G$, where $\left(u_{1}, u_{2}, \ldots, u_{k}\right.$, $\left.u_{-1}, u_{-2}, \ldots, u_{-k}\right)$ are the neighbours of $v$ in clockwise order around $v$. Let $F_{i}(v)$ denote the closed infinite wedge centred at $v$ (but not including $v$ ), which is bounded by the ray $\overrightarrow{v u_{i}}$ and the ray that is opposite to the ray $\overrightarrow{v u_{-i}}$. As illustrated in Figure 2(a), we say that $v$ has the fan property if:

$-F_{i}(v) \cap F_{j}(v)=\emptyset$ for all distinct $i, j \in I$,

- there are exactly two edges of each colour incident to $v$, and

- the edges $v u_{i}$ and $v u_{-i}$ receive the same colour for all $1 \leq i \leq k$.

We proceed by induction on $|V(G)|$ with the hypothesis: "every $2 k$-tree $G$ has a geometric drawing with thickness $k$; moreover, if $|V(G)| \geq 2 k+2$, then every $2 k$-simplicial vertex $v$ of $G$ has the fan property." Let $G$ be a $2 k$-tree. Apply Lemma 8 to $G$. 

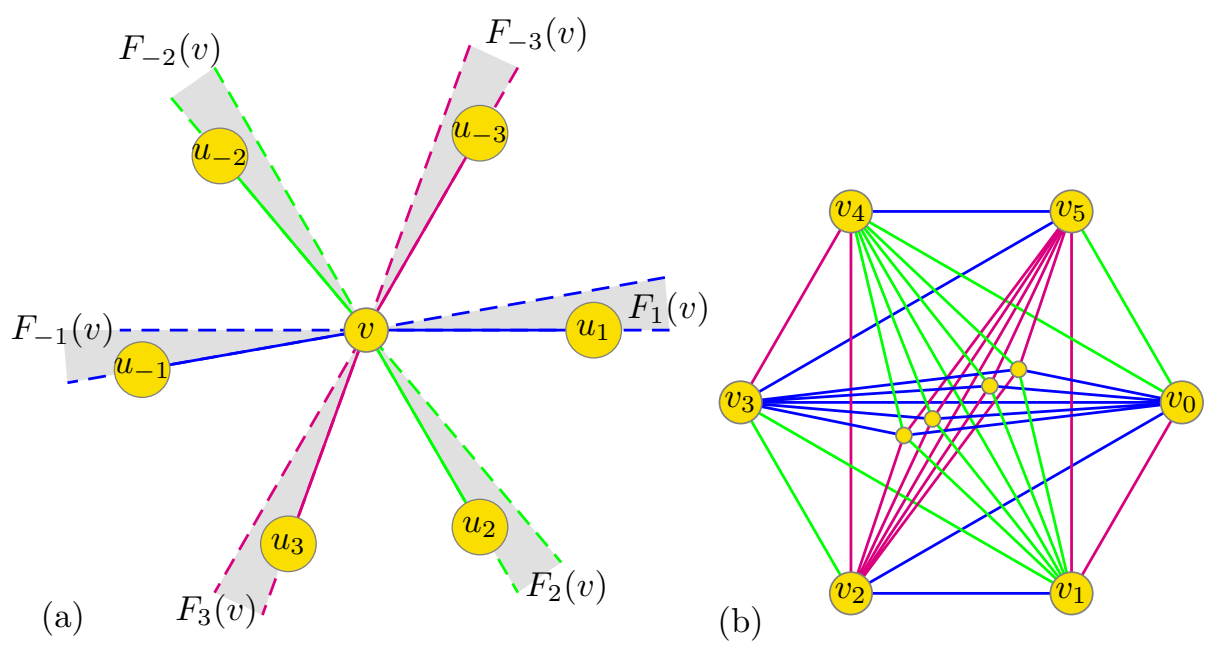

Fig. 2. Proof of Theorem 4. (a) the fan property, (b) the base case

First suppose that Lemma 8 gives a (possibly empty) independent set $S \subseteq$ $V(G)$ of $2 k$-simplicial vertices in $G$ such that $G \backslash S=K_{2 k}$. Say $V(G \backslash S)=$ $\left\{v_{0}, v_{1}, \ldots, v_{2 k-1}\right\}$. Position $v_{0}, v_{1}, \ldots, v_{2 k-1}$ evenly spaced on a circle in the plane, and in this order. The edges of $G \backslash S$ can be $k$-coloured using the standard book embedding of $K_{2 k}$ with thickness $k$, where each edge $v_{\alpha} v_{\beta}$ is coloured $\left\lfloor\frac{1}{2}((\alpha+\beta) \bmod 2 k)\right\rfloor$. Each colour class forms a plane zig-zag pattern. For each vertex $w \in S$ and for all $0 \leq i \leq k-1$, colour the edges $w v_{i}$ and $w v_{k+i}$ by $i$. As illustrated in Figure 2(b), position the vertices in $S$ in a small enough region near the centre of the circle so that monochromatic edges do not cross, each $w \in S$ has the fan property, and $V(G)$ is in general position. If $|V(G)| \geq 2 k+2$, then no vertex in $\left\{v_{0}, v_{1}, \ldots, v_{2 k-1}\right\}$ is $2 k$-simplicial in $G$. Therefore, each $2 k$-simplicial vertex of $G$ is in $S$, and thus has the fan property.

Now suppose that Lemma 8 gives a nonempty independent set $S \subseteq V(G)$ of $2 k$-simplicial vertices in $G$ and a vertex $v \in V(G) \backslash S$, such that $v$ is $2 k$-simplicial in the $k$-tree $G \backslash S$. If $|V(G) \backslash S| \geq 2 k+2$, then by induction, there is a geometric drawing of $G \backslash S$ with thickness $k$, in which $v$ has the fan property. Otherwise, $G \backslash S=K_{2 k+1}$ and thus the set $S^{\prime}=\{v\}$ is an independent set of $2 k$-simplicial vertices in $G \backslash S$ such that $(G \backslash S) \backslash S^{\prime}=K_{2 k}$. Thus by the construction given above, there is a geometric drawing of $G \backslash S$ with thickness $k$, in which $v$ has the fan property.

Say $N_{G \backslash S}(v)=\left(u_{1}, u_{2}, \ldots, u_{k}, u_{-1}, u_{-2}, \ldots, u_{-k}\right)$ in clockwise order about $v$. Without loss of generality, the edges $v u_{i}$ and $v u_{-i}$ are coloured $i$, for all $1 \leq i \leq k$. Choose a small enough disc $D_{\epsilon}$ centred at $v$ such that:

(a) the only vertices in $R_{\epsilon}$ are $N_{G \backslash S}[v]$,

(b) every edge of $G \backslash S$ that intersects $D_{\epsilon}$ is incident to $v$ (as illustrated in Figure [3), and 


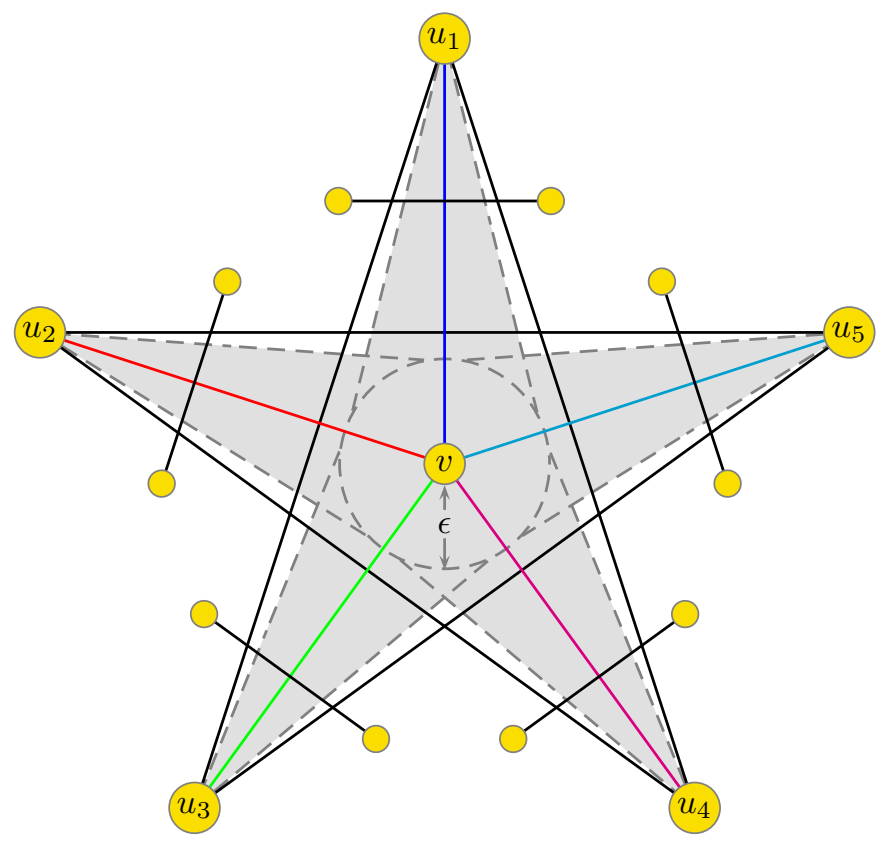

Fig. 3. The 'empty' disc $D_{\epsilon}$

(c) should a vertex whose neighbourhood is $\left\{u_{1}, u_{2}, \ldots, u_{k}, u_{-1}, u_{-2}, \ldots, u_{-k}\right\}$ be placed in $D_{\epsilon}$, then it would have the fan property.

By Lemma 8, for every vertex $w \in S$, there is exactly one $i \in I$ for which $N_{G}(w)=N_{G \backslash S}[v] \backslash\left\{u_{i}\right\}$. Let $S_{i}:=\left\{w \in S: N_{G}(w)=N_{G \backslash S}[v] \backslash\left\{u_{i}\right\}\right\}$ for all $i \in I$. Two vertices in $S_{i}$ have the same neighbourhood in $G$. For all $i \in I$, choose one vertex $x_{i} \in S_{i}$ (if any). We will first draw $x_{i}$ for all $i \in I$. Once that is completed, we will draw the remaining vertices in $S$.

As illustrated in 4 , for all $i \in I$, colour the edge $x_{i} v$ by $|i|$, and colour the edge $x_{i} u_{j}$ by $|j|$ for all $j \in I \backslash\{i\}$. Now in a drawing of $G$, for each $i \in I, F_{i}\left(x_{i}\right)$ is the closed infinite wedge bounded by the ray $\overrightarrow{x_{i} v}$ and the ray that is opposite to $\overrightarrow{x_{i} u_{-i}}$, and $F_{-i}\left(x_{i}\right)$ is the closed infinite wedge bounded by the ray $\overrightarrow{x_{i} u_{-i}}$ and the ray that is opposite to $\overrightarrow{x_{i} v}$. Observe that in a drawing of $G$, if $x_{i} \in F_{-i}(v)$ for all $i \in I$, then $v \notin F_{\ell}\left(x_{i}\right)$ for all $\ell \neq i$. Therefore, for $i \in I$ in some arbitrary order, each vertex $x_{i}$ can initially be positioned on the line-segment $\overline{v u_{-i}} \cap\left(D_{\epsilon} \backslash\{v\}\right)$, so that $x_{i} \notin \bigcup\left\{F_{\ell}\left(x_{j}\right): \ell \in I \backslash\{j\}\right\}$ for every $j \in I$. This is possible by the previous observation, since there is always a point close enough to $v$ where $x_{i}$ can be positioned, so that $x_{i} \notin \bigcup\left\{F_{\ell}\left(x_{j}\right): \ell \in I \backslash\{j\}\right\}$ for all the vertices $x_{j}$ that are drawn before $x_{i}$. Observe that each vertex $x_{i}$ has the fan property in the thus constructed illegal drawing.

Now we move each vertex $x_{i}$ just off the edge $v u_{-i}$ to obtain a legal drawing. In particular, move each $x_{i}$ by a small enough distance $\epsilon^{\prime}$ into $F_{-i}(v)$, so that 

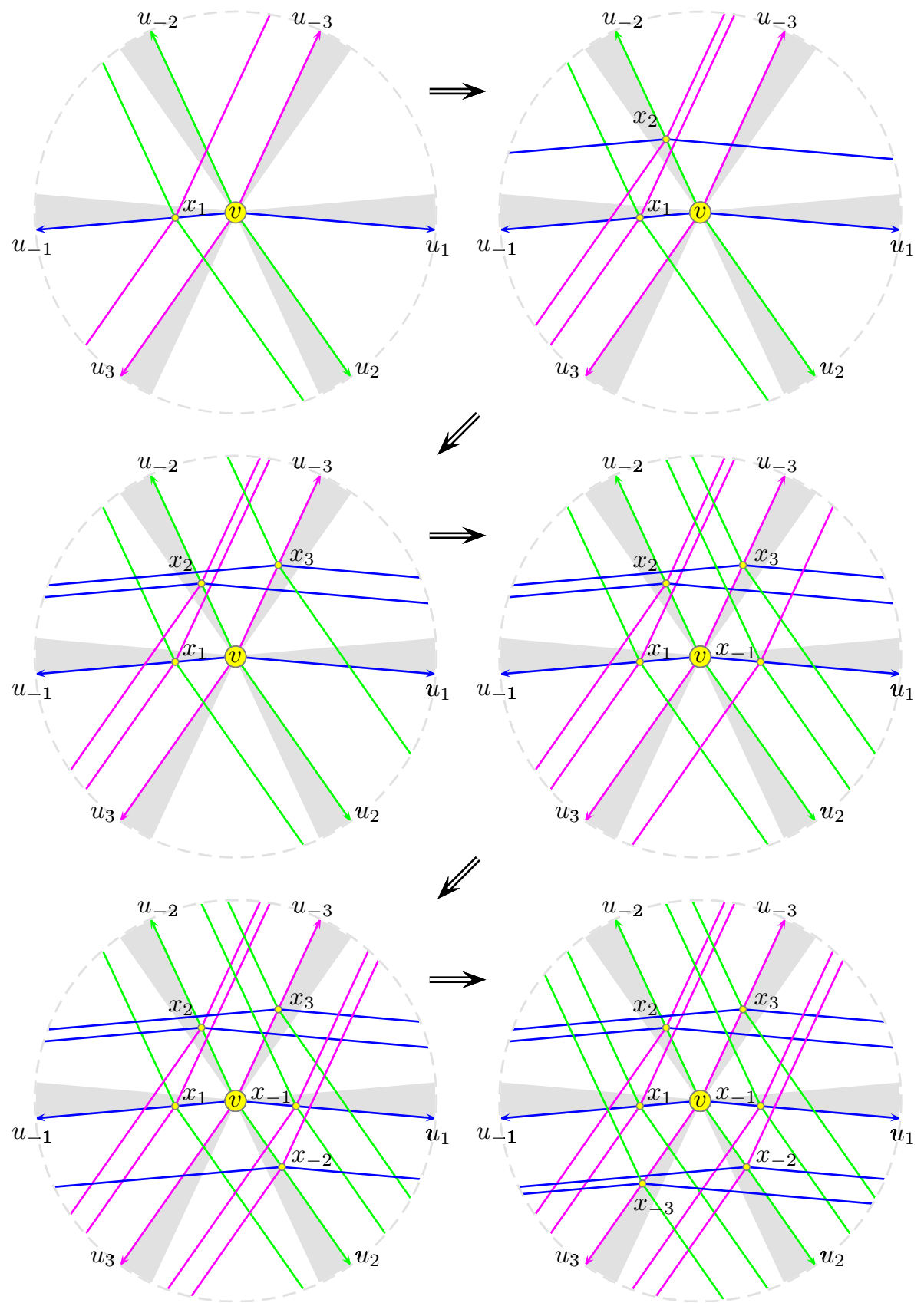

Fig. 4. Placing each $x_{i}$ on the edge $v u_{-i}$; the circle $D_{\epsilon}$ is chosen small enough so that the edges incident with $u_{i}$ are almost parallel 
$F_{i}\left(x_{i}\right)$ does not contain the vertex $x_{j}$, for all $j \in I \backslash\{i,-i\}$. This implies that for all distinct $i, j \in I$ with $i \neq-j$, we have that $x_{j} \notin F_{\ell}\left(x_{i}\right)$ for all $\ell \in I$.

To prove that monochromatic edges do not cross, we distinguish four types of edges coloured $i$, where $1 \leq i \leq k$ :

1. edges of $G \backslash S$ coloured $i$,

2. the edges $x_{i} v$ and $x_{-i} v$,

3. edges $x_{j} u_{i}$ for some $j \in I \backslash\{i\}$, and

4. edges $x_{\ell} u_{-i}$ for some $\ell \in I \backslash\{-i\}$.

First we prove that no type-(1) edge is involved in a monochromatic crossing. No two type-(1) edges cross by induction. Since a type-(2) edge is contained in $D_{\epsilon}$, by (b) in the choice of $\epsilon$, type-(1) and type-(2) edges do not cross. Suppose that a type-(1) edges $e$ crosses a type-(3) or type-(4) edge. By (a) in the choice of $\epsilon, e$ would also cross $v u_{i}$. Since $v u_{i}$ is coloured $i$, by induction applied to $G \backslash S$, $e$ is not coloured $i$.

The two type-(2) edges do not cross since they are both incident to $v$. Type(3) edges do not cross since they are all incident to $u_{i}$. Type-(4) edges do not cross since they are all incident to $u_{-i}$.

Suppose that a type-(2) edge $x_{i} v$ crosses a type-(3) edge $x_{j} u_{i}$ for some $j \in$ $I \backslash\{i\}$. By construction, $x_{i} \in F_{-i}(v)$ and $x_{j} \notin F_{-i}(v)$. Therefore, if $x_{j} u_{i}$ crosses $x_{i} v$, then $x_{j} u_{i}$ also crosses the edge $v u_{-i}$, which is a type-(1) edge of colour $|i|$. Thus this type of crossing was ruled out when type-(1) edges were considered. Now suppose that a type-(2) edge $x_{-i} v$ crosses a type-(3) edge $x_{j} u_{i}$ for some $j \in I \backslash\{i\}$. Then $x_{j} \in F_{-i}\left(x_{-i}\right)$, which contradicts the placement of $x_{j}$. Thus no type-(2) edge crosses a type-(3) edge. By symmetry, no type-(2) edge crosses a type-(4) edge.

If a type-(3) edge $x_{-i} u_{i}$ crosses a type-(4) edge $x_{\ell} u_{-i}$ (for some $\ell \in I \backslash\{-i\}$ ), then $x_{\ell} u_{-i}$ also crosses the edge $v u_{i}$, which is a type-(1) edge coloured $|i|$. Thus this type of crossing was ruled out when type-(1) edges were considered. By symmetry, a type-(4) edge $x_{i} u_{-i}$ does not cross a type-(3) edge $x_{\ell} u_{i}$ (for all $\ell \in I \backslash\{i\}$ ). Finally, if a type-(3) edge $x_{j} u_{i}$ (for some $j \in I \backslash\{i,-i\}$ ) crosses a type-(4) edge $x_{\ell} u_{-i}$ (for some $\ell \in I \backslash\{-i, i\}$ ), then $x_{\ell} \in F_{i}\left(x_{j}\right)$ and $x_{j} \in F_{-i}\left(x_{\ell}\right)$, contradicting our placement of $x_{\ell}$ or $x_{j}$. Thus type-(3) edges do not cross type(4) edges.

Each vertex $z \in S_{i} \backslash\left\{x_{i}\right\}$ can be drawn in a small enough region around $x_{i}$, and every edge $z u_{j}$ coloured with the same colour as $x_{i} u_{j}$, so that $z$ has fan property and monochromatic edges do not cross.

It remains to prove that each $2 k$-simplicial vertex of $G$ has the fan property whenever $|V(G)| \geq 2 k+2$. By construction that is true for all $2 k$-simplicial vertices of $G$ that are in $S$. The remaining $2 k$-simplicial vertices of $G$ are also $2 k$-simplicial in the $2 k$-tree $G \backslash S$. If $|V(G) \backslash S| \geq 2 k+2$, then by induction, the invariant is also maintained for all $2 k$-simplicial vertices of $G$ that are not in $S$. If $G \backslash S$ is $K_{2 k+1}$, then by Lemma 8 (d), there is no $2 k$-simplicial vertex of $G$ in $G \backslash S$. Thus the invariant is maintained. 


\section{References}

[1] Yasukazu Aoki. The star-arboricity of the complete regular multipartite graphs. Discrete Math., 81(2):115-122, 1990.

[2] Frank R. Bernhart and Paul C. Kainen. The book thickness of a graph. J. Combin. Theory Ser. B, 27(3):320-331, 1979.

[3] Emilio Di Giacomo, Walter Didimo, Giuseppe Liotta, and Stephen K. Wismath. Book embeddings and point-set embeddings of series-parallel digraphs. In Michael T. Goodrich and Stephen G. Kobourov, eds., Proc. 10th International Symp. on Graph Drawing (GD '02), vol. 2528 of Lecture Notes in Comput. Sci., pp. 162-173. Springer, 2002.

[4] Guoli Ding, Bogdan Oporowski, Daniel P. Sanders, and Dirk Vertigan. Partitioning graphs of bounded tree-width. Combinatorica, 18(1):1-12, 1998.

[5] Vida Dujmović And David R. Wood. On linear layouts of graphs. Discrete Math. Theor. Comput. Sci., 6(2):339-358, 2004.

[6] Vida Dujmović And David R. Wood. Graph treewidth and geometric thickness parameters. arXiv.org:math.CO/0503553, 2005.

[7] Joseph L. Ganley and Lenwood S. Heath. The pagenumber of $k$-trees is O(k). Discrete Appl. Math., 109(3):215-221, 2001.

[8] Richard K. Guy. Outerthickness and outercoarseness of graphs. In Proc. British Combinatorial Conf., vol. 13 of London Math. Soc. Lecture Note Ser., pp. 57-60. Cambridge Univ. Press, 1974.

[9] John H. Halton. On the thickness of graphs of given degree. Inform. Sci., 54(3):219-238, 1991.

[10] Paul C. Kainen. Thickness and coarseness of graphs. Abh. Math. Sem. Univ. Hamburg, 39:88-95, 1973.

[11] Petra Mutzel, Thomas Odenthal, and Mark Scharbrodt. The thickness of graphs: a survey. Graphs Combin., 14(1):59-73, 1998.

[12] Crispin St. J. A. Nash-Williams. Decomposition of finite graphs into forests. J. London Math. Soc., 39:12, 1964.

[13] JÁnos Pach and Rephael Wenger. Embedding planar graphs at fixed vertex locations. Graphs Combin., 17(4):717-728, 2001.

[14] S. Rengarajan and C. E. Veni Madhavan. Stack and queue number of 2 trees. In Ding-Zhu Du And Ming Li, eds., Proc. 1st Annual International Conf. on Computing and Combinatorics (COCOON '95), vol. 959 of Lecture Notes in Comput. Sci., pp. 203-212. Springer, 1995.

[15] Minalis Yannakakis. Embedding planar graphs in four pages. J. Comput. System Sci., 38:36-67, 1986. 